\title{
NUMERICAL VERIFICATION AND COMPARISON OF ERROR OF ASYMPTOTIC EXPANSION SOLUTION OF THE DUFFING EQUATION
}

\author{
Jianping Cai ${ }^{1,2, *}$, Shuhui Chen ${ }^{1}$, Cuihong Yang ${ }^{3}$ \\ ${ }^{1}$ Department of Applied Mechanics and Engineering, Zhongshan University, \\ Guangzhou 510275, China \\ ${ }^{2}$ Department of Mathematics, Zhangzhou Teachers College, Zhangzhou 363000, China \\ ${ }^{3}$ Department of Mathematics, Central China Normal University, Wuhan 430079, China \\ mathcai@hotmail.com
}

\begin{abstract}
A numerical order verification technique is applied to demonstrate that the asymptotic expansions of solutions of the Duffing equation obtained respectively by the Lindstedt-Poincaré(LP) method and the modified Lindstedt-Poincaré(MLP) method are uniformly valid for small parameter values. A numerical comparison of error shows that the MLP method is valid whereas the LP method is invalid for large parameter values.
\end{abstract}

Keywords- nonlinear oscillation, perturbation method, asymptotic expansion solution, numerical verification

\section{INTRODUCTION}

The Duffing equation

$$
\ddot{u}+\omega_{0}^{2} u+\varepsilon u^{3}=\varepsilon p \cos \Omega t
$$

has been used to model a number of mechanical and electrical systems [1]. The differential equation that describes this oscillator has a cubic nonlinearity, and it has been named after the studies of G. Duffing in the 1930's. Traditional perturbation methods, such as the Lindstedt-Poincaré(LP) method, the multiple scale method and the harmonic balance method, are powerful tools for obtaining approximate solutions of the Duffing equation as well as other nonlinear equations. Asymptotic expansion solutions by perturbation methods are formally in the form of a power series of small parameter $\varepsilon$ and are valid only for small value of $\varepsilon$ [2]. A modified Lindstedt-Poincaré(MLP) method $[3,4]$ was proposed to obtain asymptotic expansion solutions of the Duffing equation, which works not only for small parameter values but also for large parameter values of $\varepsilon$. The essential idea of the MLP method is to transform the parameter $\varepsilon$ into a new parameter $\alpha=\alpha(\varepsilon)$, which is defined such that the value of $\alpha$ is always kept small regardless of the magnitude of the original parameter $\varepsilon$. When an asymptotic expansion is formally constructed, it is important to verify that it accurately approximates the exact solution and that the error in the expansion behaves asymptotically as expected. Typically, asymptotic solutions for a few specific values of $\varepsilon$ are chosen to show that the error between the asymptotic solution and exact (numerical) solution is relatively small. However, so few comparisons are sometimes insufficient to demonstrate that the asymptotic expansion is uniformly valid, which means the numerical error of the truncated asymptotic expansion is of the same order of 
magnitude with respect to the expansion parameter as the terms neglected. Although the quantitative error may be small, it does not become small at the rate expected $[2,5]$. Therefore, one needs to further verify that the solution is indeed asymptotically accurate to the order to which it is constructed. In this paper, a numerical order verification technique, first proposed by Bosley [5], will be applied to demonstrate that the asymptotic expansions of solutions of the Duffing equation are uniformly valid up to the third order for small values of parameter $\varepsilon$. The order of the asymptotic expansion solutions of free vibration of the Duffing equation has been verified in Ref.[6], but we note that the reversion method is adopted there and the consequent expansions contain the secular term $\varepsilon t \sin t$, which are effective only for small values of $\varepsilon t$. In this paper, the use of the LP method and the MLP method can overcome this defect. Furthermore, instead of evaluating the asymptotic solution at one fixed point $t=t_{0}$ in Refs.[5-9] or finite fixed points $t=t_{i}(i=1,2, \cdots, m)$ in Ref.[10], maximum absolute error on an interval $[0, T]$ is introduced in this paper to give a more comprehensive evaluation of the error between the asymptotic and numerical solutions. When estimating numerical errors due to time evolution, it is more correct to use the maximum error on the time domain in engineering applications. Finally, a numerical comparison of the error of the LP method with that of the MLP method shows that the MLP method works also for large values of $\varepsilon$ whereas the LP method is invalid.

\section{ASYMPTOTIC EXPANSIONS OF SOLUTIONS}

Consider the harmonically excited vibrations of the Duffing equation

$$
\begin{aligned}
& \ddot{u}+\omega_{0}^{2} u+\varepsilon u^{3}=\varepsilon p \cos \Omega t, \\
& u(0)=a, \dot{u}(0)=0,
\end{aligned}
$$

where $\Omega$ is the forcing frequency, $p$ is the forcing amplitude. Taking a time transformation $\tau=\Omega t$, Eq.(1) becomes

$$
\Omega^{2} u^{\prime \prime}+\omega_{0}^{2} u+\varepsilon u^{3}=\varepsilon p \cos \tau
$$

Following the procedure of the classical LP method [2], the first four terms of the approximate solution of fundamental resonance can be worked out as follows

$$
u_{\text {asym }}(t)=u_{0}(t)+\varepsilon u_{1}(t)+\varepsilon^{2} u_{2}(t)+\varepsilon^{3} u_{3}(t),
$$

where

$$
\begin{aligned}
& \Omega=\omega_{0}+\left(\frac{3 a^{2}}{8 \omega_{0}}-\frac{p}{2 a \omega_{0}}\right) \varepsilon-\frac{1}{256 a^{2} \omega_{0}^{3}}\left(21 a^{6}-44 a^{3} p+32 p^{2}\right) \varepsilon^{2} \\
& +\frac{1}{2048 a^{3} \omega_{0}^{5}}\left(81 a^{9}-221 a^{6} p+236 a^{3} p^{2}-128 p^{3}\right) \varepsilon^{3}, \\
& u_{0}(t)=a \cos \tau \\
& u_{1}(t)=\frac{a^{3}}{32 \omega_{0}^{2}}(\cos 3 \tau-\cos \tau), \\
& u_{2}(t)=\frac{1}{1024 \omega_{0}^{4}}\left(a^{5} \cos 5 \tau+\left(36 a^{2} p-24 a^{5}\right) \cos 3 \tau+\left(23 a^{5}-36 a^{2} p\right) \cos \tau\right),
\end{aligned}
$$




$$
\begin{array}{r}
u_{3}(t)=\frac{1}{98304 \omega_{0}^{6}}\left(3 a^{7} \cos 7 \tau+\left(208 a^{4} p-144 a^{7}\right) \cos 5 \tau\right. \\
+\left(1782 a^{7}-5076 a^{4} p+3888 a p^{2}\right) \cos 3 \tau \\
\left.+\left(-1641 a^{7}+4868 a^{4} p-3888 a p^{2}\right) \cos \tau\right) .
\end{array}
$$

Similarly, following the procedure of the MLP method [4], the first four terms of the approximate solution of fundamental resonance of Eq.(3) can be worked out as follows

$$
u_{\text {asym }}(t)=u_{0}(t)+\alpha u_{1}(t)+\alpha^{2} u_{2}(t)+\alpha^{3} u_{3}(t),
$$

where

$$
\alpha=\frac{\varepsilon \omega_{1}}{\omega_{0}^{2}+\varepsilon \omega_{1}} \text { with } \omega_{1}=\frac{3}{4} a^{2}-\frac{p}{a},
$$

and

$$
\begin{aligned}
\Omega^{2}= & \frac{\omega_{0}^{2}}{1-\alpha}\left(1-\frac{a}{128 \omega_{1}^{2}}\left(3 a^{3}+4 p\right) \alpha^{2}+\frac{a p}{256 \omega_{1}^{3}}\left(\omega_{1}-2 a^{2}\right) \alpha^{3}+O\left(\alpha^{4}\right)\right) \\
u_{0}(t)= & a \cos \tau \\
u_{1}(t)= & \frac{a^{3}}{32 \omega_{1}}(\cos 3 \tau-\cos \tau) \\
u_{2}(t)= & \frac{1}{1024 \omega_{1}^{2}}\left(a^{5} \cos 5 \tau+4 a^{2} p \cos 3 \tau-a^{2}\left(4 p+a^{3}\right) \cos \tau\right) \\
u_{3}(t)= & \frac{1}{98304 \omega_{1}^{3}}\left(3 a^{7} \cos 7 \tau+16 a^{4} p \cos 5 \tau+\left(54 a^{7}+48 a^{2} p\left(3 a^{2}-\omega_{1}\right)\right) \cos 3 \tau\right. \\
& \left.+\left(57 a^{7}+160 a^{4} p-48 a^{2} p \omega_{1}\right) \cos \tau\right) .
\end{aligned}
$$

\section{NUMERICAL ORDER VERIFICATION OF ASYMPTOTIC EXPANSIONS}

We first give a brief introduction to the Bosley's technique [5]. Assume that the asymptotic expansion solution of a nonlinear equation is

$$
u_{\text {asym }}(t, \varepsilon)=u_{0}(t)+\varepsilon u_{1}(t)+\varepsilon^{2} u_{2}(t)+\cdots+\varepsilon^{N} u_{N}(t)+O\left(\varepsilon^{N+1}\right) .
$$

The absolute error between the asymptotic solution and the exact solution is

$$
\begin{aligned}
\text { Error } & =E_{N}(t, \varepsilon)=\left|u_{\text {exact }}(t, \varepsilon)-u_{\text {asym }}(t, \varepsilon)\right| \\
& =\left|u_{\text {exact }}(t, \varepsilon)-\sum_{n=0}^{N} \varepsilon^{n} u_{n}(t)\right|=O\left(\varepsilon^{N+1}\right)=K \varepsilon^{N+1},
\end{aligned}
$$

where $K$ is a constant. Taking the logarithm of both sides of Eq.(18) yields

$\log E_{N}=\log K+(N+1) \log \varepsilon$.

If $E_{N}$ is of order $O\left(\varepsilon^{N+1}\right)$ for a fixed time $t=t_{0}$ and for small values of $\varepsilon$, the value of $\log E_{N}$ as a function of $\log \varepsilon$ should be linear with slope $N+1$. Therefore, when we graph $\log E_{N}$ versus $\log \varepsilon$ for different values of $\varepsilon$, these points should be nearly on a 
line and the linear equation that interpolates these points using a linear least-squares fit should have slope $N+1$.

The error of Eq.(18) is evaluated at a fixed point $t=t_{0}$ in Refs.[5-9]. We think it is partial because the errors are different at different points, namely, the error may be small at one point but large at the other points. In this paper, maximum absolute error on an interval $[0, T]$ is introduced as

$$
\text { Maximum Error }=\max E_{N}(t, \varepsilon)=\max _{t \in[0, T]}\left\{E_{N}(t, \varepsilon)\right\},
$$

which can give a comprehensive estimation of difference between the exact solution and the asymptotic solution over the domain of interest. Eq.(19) can be numerically approximated by

$$
\text { Maximum Error }=\max E_{N}(t, \varepsilon)=\max \left\{E_{N}\left(t_{i}, \varepsilon\right), i=1,2, \cdots, m\right\}
$$

where $t_{i}$ are fixed points in the interval $[0, T]$ and $m$ is sufficiently large integer.

In the following examples, the values of parameters in Eqs.(1), (2), (19) and (20) are assumed to be $\omega_{0}=1, a=4, p=16, T=10, t_{i}=0.05 i, i=1,2, \cdots, 200$. To verify the order of asymptotic expansion (4) obtained by the LP method, we first find the numerical solutions of Eqs.(1) and (2) with $\varepsilon$ starting from 0.001 and ending at 0.03 by a step size 0.001 . Next, we evaluate the asymptotic expansion (4) at the same values of $\varepsilon$ and $t_{i}$ as the numerical solutions for $N=0,1,2$ and 3 respectively. In Fig. 1 we plot the values of the error at these 30 points, namely, $\log E_{0}, \log E_{1}, \log E_{2}$ and $\log E_{3}$ as functions of $\log \varepsilon$. The exact solution $u_{\text {exact }}(t, \varepsilon)$ in Eq.(18) is replaced by the numerical solution. For $N=0,1,2$ and 3, the least-squares fit of these data is used to determine respectively the slopes $1.00252,1.96014,2.95284$ and 3.94723 , whose relative errors are less than $2 \%$, compared with the theoretical slopes $N+1=1,2,3$ and 4 , respectively. So we can conclude that the asymptotic solution of the Duffing equation obtained by the LP method is indeed uniformly valid for small parameter values. In this paper, the computer algebra system Mathematica is applied to implement relative calculations and plots.

Similarly, the verification of the order of asymptotic expansion (10) with (11)-(16) obtained by the MLP method is shown in Fig.2, where $\alpha$ starts from 0.005(0.0006 for $\varepsilon$ ) and ends at $0.1(0.0139$ for $\varepsilon)$ by a step size 0.0025 . For $N=0,1,2$ and 3 , the least-squares fit of these data is used to determine respectively the slopes 1.0001, 2.05442, 2.99075 and 4.00381, whose relative errors are less than $2.7 \%$, compared with the theoretical slopes $N+1=1,2,3$ and 4, respectively. Thus we can conclude that the asymptotic solution of the Duffing equation obtained by the MLP method is indeed uniformly valid for small parameter values.

\section{NUMERICAL COMPARISON OF THE MLP METHOD WITH THE LP METHOD}

Now we show a numerical comparison of the MLP method with the LP method. For simplicity, we take only the third order approximation as an example. 
In Fig.3 we plot $E_{3}$ versus $\varepsilon$ of the asymptotic expansion (4) with (5)-(9) obtained by the LP method, where $\varepsilon$ starts from 0.5 and ends at 2.5 by a step size 0.05 . When $\varepsilon>0.5$, the LP method is unacceptable because the maximum absolute error $E_{3}$ is larger than 21 and increases rapidly as $\varepsilon$ increases. For $\varepsilon=2.5$, the error is as large as 2824.

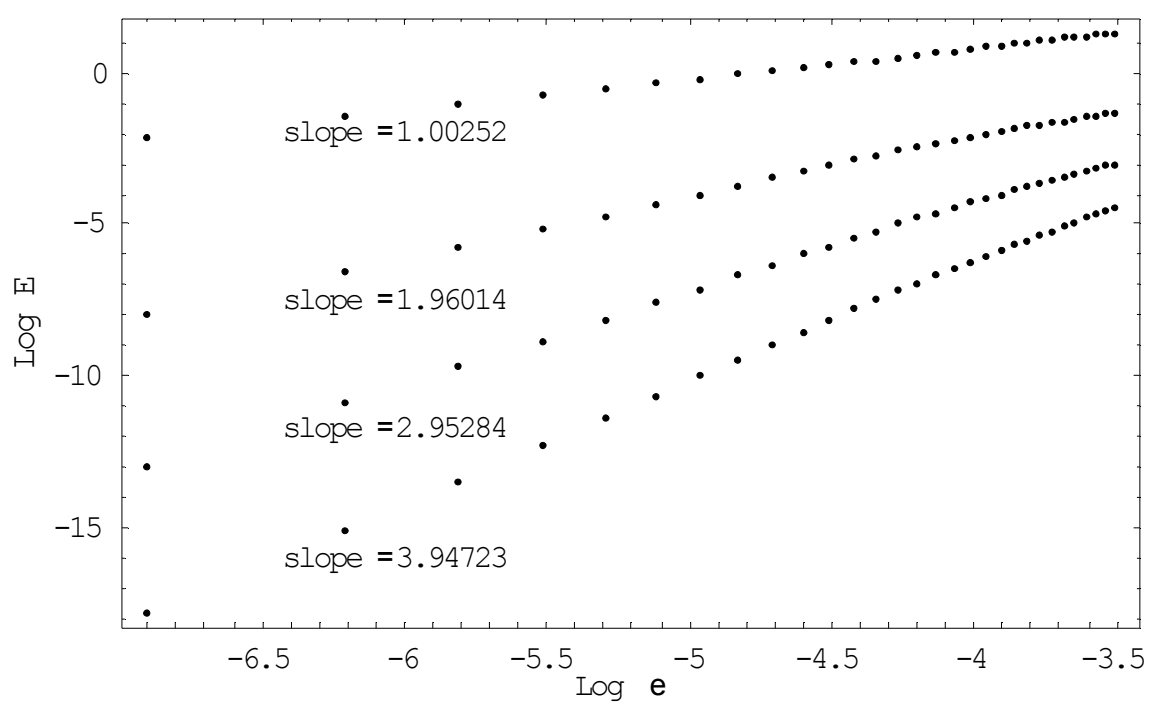

Fig.1 Order verification of the asymptotic expansion (4) obtained by the LP method

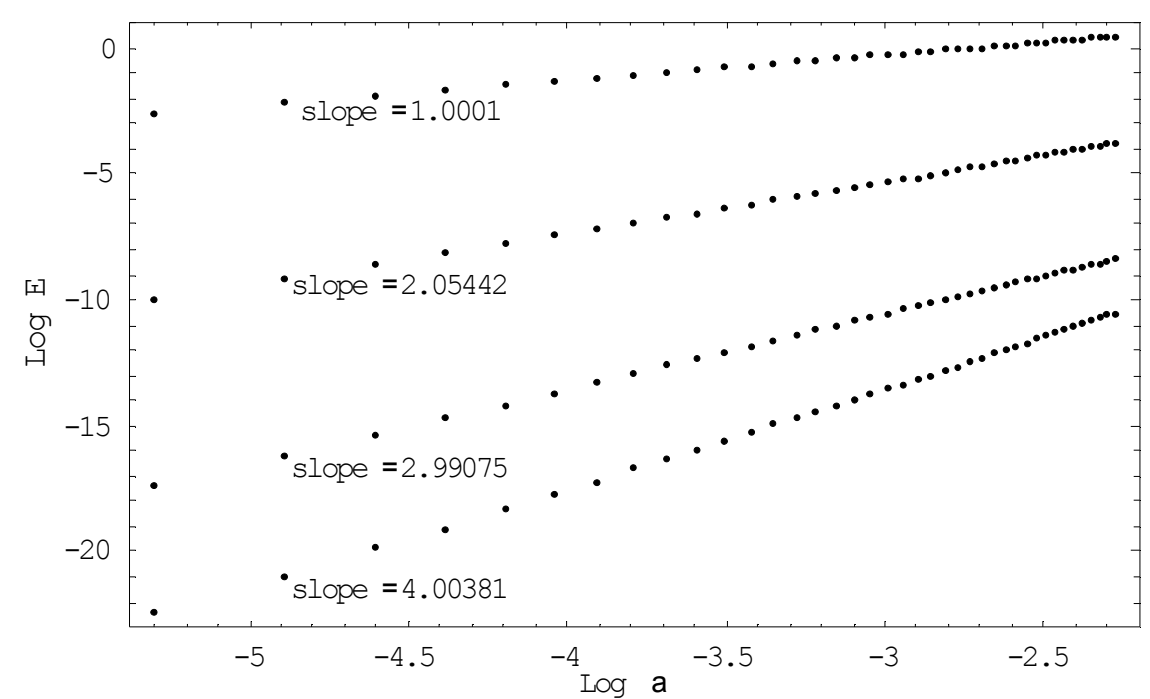

Fig.2 Order verification of the asymptotic expansion (10) obtained by the MLP method

In Fig. 4 we plot $E_{3}$ versus $\varepsilon$ of the asymptotic expansion (10) with (11)-(16) obtained by the MLP method, where the parameters are the same as above. Note that the maximum absolute error $E_{3}$ is only 0.074 (corresponding relative error is $1.85 \%$ ) for all $\varepsilon$ starting from 0.5 and ending at 2.5 . 


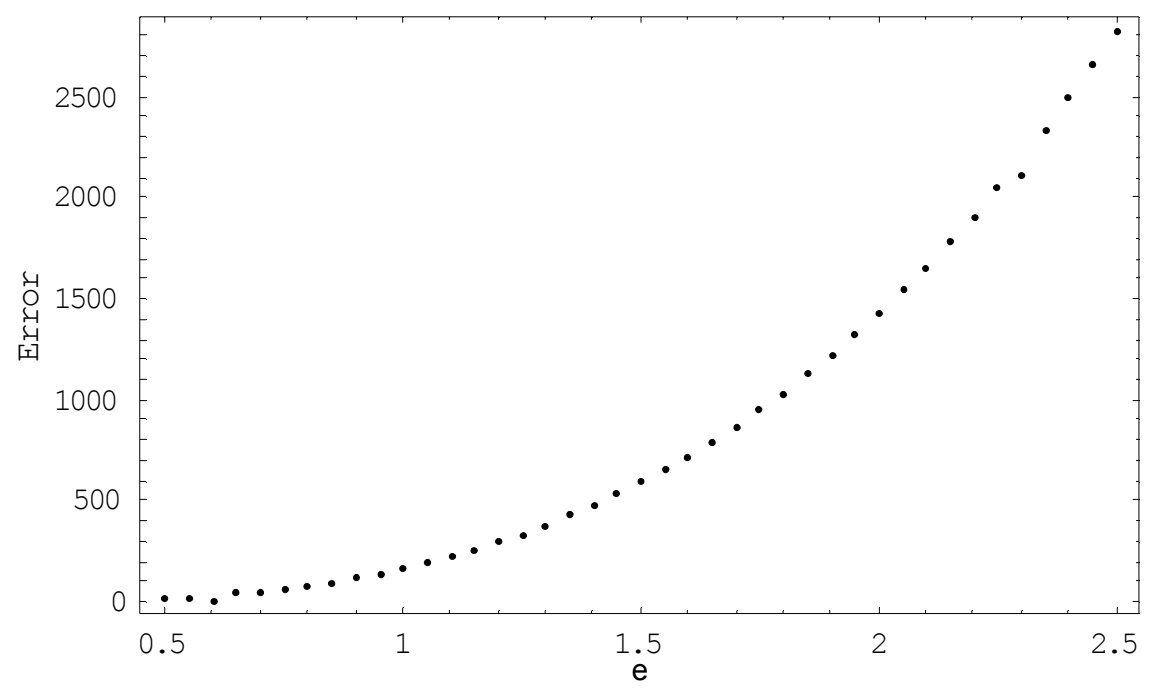

Fig.3 Error of the expansion (4) obtained by the LP method for large parameter values of $\varepsilon$

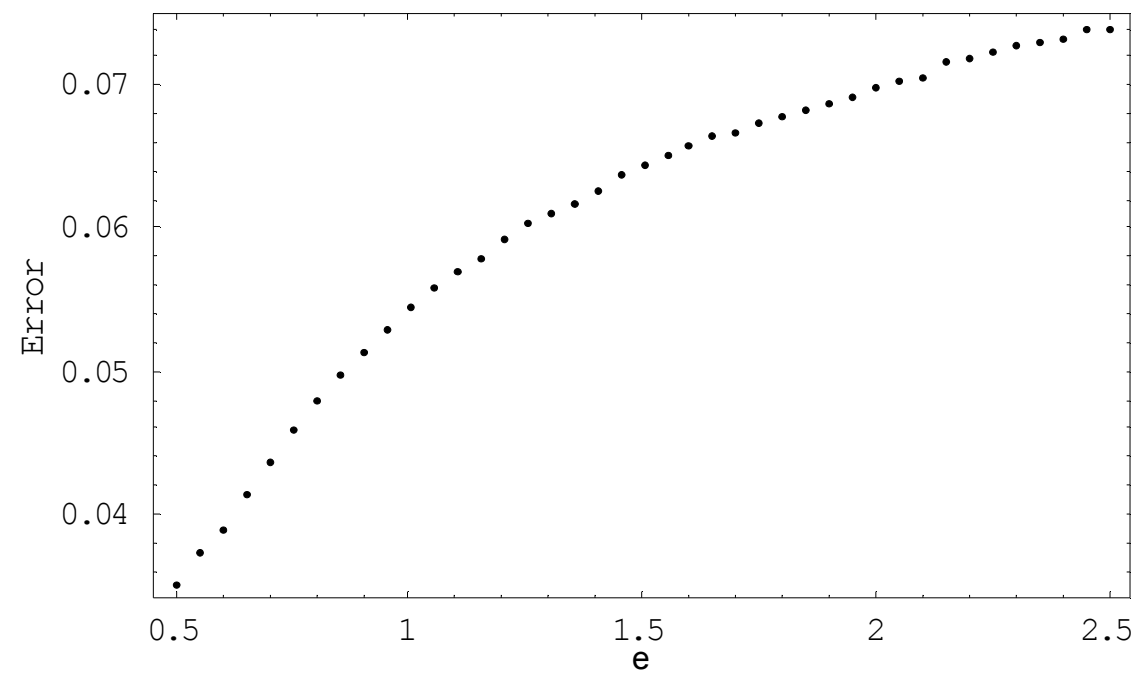

Fig.4 Error of the expansion (10) obtained by the MLP method for large parameter values of $\varepsilon$

So, for large parameter values of $\varepsilon$, the MLP method is valid whereas the LP method is invalid.

\section{CONCLUSIONS}

The asymptotic expansions of solutions of the Duffing equation obtained by the LP method and the MLP method are uniformly valid for small parameter values of $\varepsilon$. For large parameter values of $\varepsilon$, the MLP method is valid whereas the LP method is invalid.

Acknowledgement- Research is supported by the National Natural Science Foundation of China under grant No.10672193, China Postdoctoral Science Foundation under grant 
No.2004036517, and the Foundation of Advanced Research Center of Zhongshan University under grant No. 06M13.

\section{REFERENCES}

1. A.H. Nayfeh and D. T. Mook, Nonlinear Oscillations, Wiley, New York, 1979.

2. A.H. Nayfeh, Introduction to perturbation methods, Wiley, New York, 1981.

3. T. D. Burton, A perturbation method for certain non-linear oscillators, International Journal of Non-Linear Mechanics, 19(5), 397-407, 1984.

4. Y.K.Cheung, S.H.Chen and S.L.Lau, A modified Lindstedt-Poincaré method for certain strongly nonlinear equations, International Journal of Non-Linear Mechanics, 26(3/4), 367-378, 1991.

5. D.L.Bosley, A technique for the numerical verification of asymptotic expansions, SIAM Review, 38(1),128-135, 1996.

6. S.A.Khuri and S.Xie. On the numerical verification of the asymptotic expansion of Duffing's equation, International Journal of Computational Mathematics, 72, 325330, 1999.

7. Elias Deeba and Shishen Xie, The asymptotic expansion and numerical verification of Van der Pol's equation, Journal of Computational Analysis and Applications, 3(2), 165-171, 2001.

8. S.A.Khuri, Numerical order verfication of the asymptotic expansion of a nonlinear differential equation arising in general relativity, Applied Mathematics and Computation, 134,147-151, 2003.

9. B.Mudavanhu and R.E.O'Malley, JR. A new renormalization method for the asymptotic solution of weekly nonlinear vector systems, SIAM Journal of Applied Mathematics, 63(2), 373-397, 2002.

10. Jianping Cai, Numerical verification of the order of the asymptotic solutions of a nonlinear differential equation, Mathematical and Computational Applications, 11(1), 85-90, 2006. 